TRANSACTIONS OF THE

AMERICAN MATHEMATICAL SOCIETY

Volume 351, Number 5, Pages 2081-2099

S 0002-9947(99)02195-9

Article electronically published on January 26, 1999

\title{
CONICAL LIMIT SET AND POINCARÉ EXPONENT FOR ITERATIONS OF RATIONAL FUNCTIONS
}

\author{
FELIKS PRZYTYCKI
}

\begin{abstract}
We contribute to the dictionary between action of Kleinian groups and iteration of rational functions on the Riemann sphere. We define the Poincaré exponent $\delta(f, z)=\inf \{\alpha \geq 0: \mathcal{P}(z, \alpha) \leq 0\}$, where

$$
\mathcal{P}(z, \alpha):=\limsup _{n \rightarrow \infty} \frac{1}{n} \log \sum_{f^{n}(x)=z}\left|\left(f^{n}\right)^{\prime}(x)\right|^{-\alpha} .
$$

We prove that $\delta(f, z)$ and $\mathcal{P}(z, \alpha)$ do not depend on $z$, provided $z$ is nonexceptional. $\mathcal{P}$ plays the role of pressure; we prove that it coincides with the Denker-Urbański pressure if $\alpha \leq \delta(f)$. Various notions of "conical limit set" are considered. They all have Hausdorff dimension equal to $\delta(f)$ which is equal to the hyperbolic dimension of the Julia set and also equal to the exponent of some conformal Patterson-Sullivan measures. In an Appendix we also discuss notions of "conical limit set" introduced recently by Urbański and by Lyubich and Minsky.
\end{abstract}

\section{INTRODUCTION}

For every Kleinian group $G$ with $\Lambda_{\mathrm{c}}(G)$ the conical limit set, $\delta(G)$ the Poincaré exponent, $\alpha(G)$ the infimum of exponents of conformal measures and HD standing for Hausdorff dimension, the following inequality holds:

$$
\operatorname{HD}\left(\Lambda_{\mathrm{c}}(G)\right)=\delta(G)=\alpha(G),
$$

and Patterson's construction gives conformal measures of exponent precisely equal to $\delta(G)$. This is a part of a beautiful theory linking these notions; see [Pa], [S1], [BJ] (and also [N]).

In iterations of rational functions conformal measures were introduced by $\mathrm{D}$. Sullivan [S2], and a general theory of conformal measures was developed by M. Denker and M. Urbański in [DU1] and [DU2]. In [DU2] the dynamical dimension of the Julia set $J=J(f)$ for a rational function $f$ was introduced and defined as follows

$$
\begin{array}{r}
\operatorname{HD}_{\text {mhyp }}(J)=\sup \{\operatorname{HD}(\mu): \mu \text { is an ergodic probability } f \text {-invariant measure } \\
\text { of positive Lyapunov exponent }\},
\end{array}
$$

where $\operatorname{HD}(\mu)$ is the infimum of Hausdorff dimensions of sets of full measure $\mu$ (mhyp abbreviates, measure hyperbolic). It happens (see Appendix 2 or [PUbook, Ch.8]

Received by the editors December 2, 1996.

1991 Mathematics Subject Classification. Primary 58F23.

Supported by Polish KBN Grant 2 P301 01307 and by the Max-Planck-Institut für Mathematik in Bonn, where the author stayed in Summer 1996. 
that $\operatorname{HD}_{\text {mhyp }}(J)=\operatorname{HD}_{\text {hyp }}(J)$, the hyperbolic dimension, which is the supremum of the Hausdorff dimensions of $f$-invariant isolated hyperbolic subsets of $J$, a notion introduced by M. Shishikura [Shi]. ( $X$ is called isolated if every trajectory $f^{j}(x), j=0,1, \ldots$, contained in a sufficiently small neighbourhood of $X$ must be contained in $X$. A compact $f$-invariant set $X \subset J$ is called hyperbolic if there exists $n>0$ such that $\left|\left(f^{n}\right)^{\prime}\right|>1$ on $X$.)

The main theorem of [DU2], [P2] (see also Appendix 2) asserts that

$$
\operatorname{HD}_{\text {mhyp }}(J)=\alpha(f),
$$

where $\alpha(f)$ is the infimum of exponents of conformal measures for $f$. Moreover a conformal measure for which this infimum is attained is constructed in [DU2], but not by the Patterson procedure. Recall that $\mu$ is called $\alpha$-conformal for $f$ (or conformal with exponent $\alpha$ ) if for every Borel set $E \in J$ on which $f$ is injective we have $\mu(f(E))=\int_{E}\left|f^{\prime}\right|^{\alpha} d \mu$.

However, a definition of a "conical Julia set" whose Hausdorff dimension would be equal to $\operatorname{HD}_{\text {mhyp }}(J)$, and also a definition, analogous to $\delta(G)$, of the Poincaré exponent and an equality similar to (0.1) were missing. In this paper we try to fill this gap.

\section{BASIC CONCEPTS}

Definition 1.1. For each rational function $f$ of degree $\geq 2$, for every $z \in \overline{\mathbb{C}}$ and every $\alpha \geq 0$ consider the following Poincaré sequence:

$$
P(z, \alpha, n):=\sum_{f^{n}(x)=z}\left|\left(f^{n}\right)^{\prime}(x)\right|^{-\alpha}
$$

We call $\alpha=\delta(f, z)$ the Poincaré exponent with respect to $z$ if $\alpha$ is the smallest number such that

$$
\limsup _{n \rightarrow \infty} \frac{1}{n} \log P(z, \alpha, n) \leq 0 .
$$

If this limsup is positive for every $\alpha$ we set $\delta(f, z)=\infty$.

Notice that $\delta(f, z)>0$. Indeed,

$$
P(z, \alpha, n) \geq \operatorname{deg}(f)^{n}\left(\sup \left|f^{\prime}\right|\right)^{-n \alpha} .
$$

Hence

$$
\frac{1}{n} \log P(z, \alpha, n) \geq \log \operatorname{deg}(f)-\alpha \log \left(\sup \left|f^{\prime}\right|\right)
$$

and so

$$
\delta(f, z) \geq \log \operatorname{deg}(f) / \log \left(\sup \left|f^{\prime}\right|\right) .
$$

Notice also that the smallest $\alpha$ in Definition 1.1 exists. This is an easy exercise (see Prop. A2.2).

The main theorem of the paper (Section 3) says that $\delta(f, z)$ as a function of $z$ is constant and attains its minimum everywhere except on a "thin" set $E$ (of Hausdorff dimension 0). We call this constant the Poincaré exponent and denote it by $\delta(f)$. Before we prove this main theorem we just write

$$
\delta(f)=\inf _{z} \delta(f, z) .
$$


One can define the pressure $\mathcal{P}(z, \alpha)=\mathcal{P}\left(f,-\alpha \log \left|f^{\prime}\right|, z\right)$ as

$$
\limsup _{n \rightarrow \infty} \frac{1}{n} \log P(z, \alpha, n) .
$$

So $\delta(f, z)$ is the first zero of $\mathcal{P}(z, \alpha)$ as a function of $\alpha$. $\mathcal{P}(z, \alpha)$, like $\delta(f, z)$, is a constant independent of $z$ except when $z \in E$, where it is not smaller. Denote this constant by $\mathcal{P}(\alpha)$. Clearly $\mathcal{P}(z, \alpha)$ and $\mathcal{P}(\alpha)$ are continuous (Appendix 2, Prop.A2.1).

In fact for $0 \leq \alpha \leq \delta(f), \mathcal{P}(\alpha)$ coincides with the pressure defined in [DU2] and also with $\sup h_{\mu}(f)-\alpha \int \log \left|f^{\prime}\right| d \mu$, where the supremum is taken over all ergodic $f$-invariant probability measures (or only measures with positive Lyapunov exponent), $h_{\mu}(f)$ being the entropy. So on the domain $0 \leq \alpha \leq \delta, \mathcal{P}(\alpha)$ is strictly decreasing. On $0 \leq \alpha<\infty \mathcal{P}(\alpha)$ is monotone decreasing and convex. We explain these facts concerning pressure in Appendix 2. They are not needed in the main course of the paper, but they are interesting because, for example, they generalize the variational principle $[\mathrm{W}]$ to the case when $-\log \left|f^{\prime}\right|$ has singularities of value $\infty$.

Definition 1.2. For each rational function $f$ and every non-exceptional $z$ (this means that " $z$ is not a superattracting periodic point for $f$ "), for every $K>0$ write

$$
\Lambda_{\mathrm{c}}(f, z, K):=\left\{x \in \overline{\mathbb{C}}: \exists x_{i} \in f^{-n_{i}}(z), n_{i} \rightarrow \infty, i=1,2, \ldots,\right.
$$

$(\forall i)$ the properties (i) and (ii) hold\},

where

$$
\begin{gathered}
\left|\left(f^{n_{i}}\right)^{\prime}\left(x_{i}\right)\right| \geq\left(1+K^{-1}\right)^{n_{i}}, \\
\operatorname{dist}\left(x, x_{i}\right) \leq K\left|\left(f^{n_{i}}\right)^{\prime}\left(x_{i}\right)\right|^{-1}
\end{gathered}
$$

(dist is the Riemann distance). Write

$$
\Lambda_{\mathrm{c}}(f, z):=\bigcup_{K>0} \Lambda_{\mathrm{c}}(f, z, K)
$$

Finally, define the conical limit set

$$
\Lambda_{\mathrm{c}}(f):=\bigcap_{z} \Lambda_{\mathrm{c}}(f, z)
$$

We do not know how $\Lambda_{\mathrm{c}}(f, z)$ depends on $z$. We conclude only a posteriori that except for $z \in E$ its dimension is constant, equal to $\delta(f)$. Therefore we think that the following concept is of interest:

Definition 1.3.

$$
\Lambda_{\mathrm{cw}}(f, z, K):=\left\{x \in \overline{\mathbb{C}}: \exists x_{i} \in f^{-n_{i}}(z), n_{i} \rightarrow \infty, i=1,2, \ldots,\right.
$$

$(\forall i)$ the properties (i) and (iii) hold\},

where

$$
\limsup _{i \rightarrow \infty} \frac{1}{n_{i}} \log \left(\operatorname{dist}\left(x, x_{i}\right) \cdot\left|\left(f^{n_{i}}\right)^{\prime}\left(x_{i}\right)\right|\right) \leq 0 .
$$

As before, we write

$$
\Lambda_{\mathrm{cw}}(f, z):=\bigcup_{K>0} \Lambda_{\mathrm{cw}}(f, z, K)
$$


Of course $\Lambda_{\mathrm{cw}}(f, z) \supset \Lambda_{\mathrm{c}}(f, z)$. In Section 3. we prove that $\Lambda_{\mathrm{cw}}(f, z)$ does not depend on $z$ except when $z \in E$. We then write $\Lambda_{\mathrm{cw}}(f)$ and call this the weak conical limit set. We show in Section 3 that for every $z$ which has a backward trajectory omitting Crit, the set of points where $f^{\prime}$ is zero, $\Lambda_{\mathrm{cw}}(f) \subset \Lambda_{\mathrm{cw}}(f, z)$.

Notice that (i), together with $x_{i} \rightarrow x$, implies that, for every $z, \Lambda_{\mathrm{cw}}(f, z) \subset J(f)$.

We remark finally that if two points $z_{1}, z_{2}$ belong to a connected open set $U$ which is disjoint from $O($ Crit $):=\bigcup_{n=1}^{\infty} f^{n}((f))$, then by bounded distortion for all branches of $f^{-n}$ on a connected open set with closure in $U$ containing $z_{1}$ and $z_{2}$ we have $\Lambda_{\mathrm{c}}\left(f, z_{1}\right)=\Lambda_{\mathrm{c}}\left(f, z_{2}\right)$, and the Poincaré exponents also coincide. This is the case for $z_{1}, z_{2}$ belonging to the same Fatou component, in particular for the basin $A_{\infty}$ of $\infty$ for $f$ a polynomial. So for polynomials one can define $\Lambda_{\mathrm{c}}(f):=$ $\Lambda_{\mathrm{c}}(f, z), \quad z \in A_{\infty}$.

It is easy to see that (iii) is equivalent to $x$ being non-deep for the filled-in Julia set, the notion introduced by $\mathrm{C}$. McMullen $[\mathrm{McM}]$.

In the next section two more concepts of the limit set will appear in a natural way in relation to an application of the Pesin theory. We call them regular and tree conical, and denote them by $\Lambda_{\text {reg }}(f)$ and $\Lambda_{\text {tc }}(f)$ respectively. In the case of polynomials we also define radial conical, which is equivalent to tree conical (in the case when the basin of $\infty$ is simply-connected). We will have

$$
\Lambda_{\mathrm{reg}}(f) \subset \Lambda_{\mathrm{tc}}(f) \subset \Lambda_{\mathrm{c}}(f) \subset \Lambda_{\mathrm{cw}}(f),
$$

and all these sets have the same Hausdorff dimension. In Appendix 3 we discuss concepts of conical limit set by Urbański [U2], [DMNU] and by Lyubich and Minsky $[\mathrm{LM}]$.

\section{Equalities of Dimensions AND EXPONENTS}

Theorem 2.1. For every $z$

$$
\operatorname{HD}\left(\Lambda_{\mathrm{c}}(f, z)\right) \leq \operatorname{HD}\left(\Lambda_{\mathrm{cw}}(f, z)\right) \leq \delta(f, z) .
$$

So $\operatorname{HD}\left(\Lambda_{\mathrm{c}}(f)\right) \leq \delta(f)$.

Proof. By the conditions (iii) and (i)

$$
\Lambda_{\mathrm{cw}}(f, z, K) \subset \bigcap_{\varepsilon>0} \bigcap_{N \geq 0} \bigcup_{f^{n}(y)=z, n>N} B\left(y, r(n, y)^{1-\varepsilon}\right),
$$

where $r(n, y):=\left|\left(f^{n}\right)^{\prime}(y)\right|^{-1}$ and the union consists only of terms satisfying $r(n, y)$ $\leq\left(1+K^{-1}\right)^{-n}$. By the definition of $\delta(f, z)$ and condition (i), for every $\alpha>$ $\bar{\delta}(f, z)$ the series $\sum_{n, y} r(n, y)^{\alpha}$ is convergent, even exponentially fast with $n$. Indeed, for $\eta>0$ arbitrarily small and all $n$ large enough we have

$$
\begin{gathered}
\sum_{y} r(n, y)^{\alpha}=\sum_{y}\left|\left(f^{n}\right)^{\prime}(y)\right|^{-\delta(f, z)}\left|\left(f^{n}\right)^{\prime}(y)\right|^{\delta(f, z)-\alpha} \\
\leq(\exp \eta n)\left(1+K^{-1}\right)^{-n(\alpha-\delta(f, z))} .
\end{gathered}
$$

Hence $\sum_{n, y} r(n, y)^{\alpha(1-\varepsilon)}<\infty$ for $\varepsilon>0$ small enough. So for the Hausdorff measure we have $\mathrm{H}_{\alpha}\left(\Lambda_{\mathrm{cw}}(f, z, K)\right)=0$.

(This is similar to the Kleinian groups case. The discs $B(y, K r(n, y))$ or $B\left(y, r(n, y)^{1-\varepsilon}\right)$ correspond to "shadows".)

Theorem 2.2. $\operatorname{HD}_{\text {mhyp }}(J) \leq \operatorname{HD}\left(\Lambda_{\mathrm{c}}(f)\right)$. 
Proof. This theorem follows from the following property, true for every $f$-invariant ergodic probability measure $\mu$ on $J$ with characteristic Lyapunov exponent $\chi_{\mu}(f)=$ $\int \log \left|f^{\prime}\right| d \mu>0$ and $\mu$-a.e. $x$ (see [PUbook]):

There exists $\eta>0$ and a sequence of integers $n_{j} \rightarrow \infty$ such that

$$
\limsup _{j \rightarrow \infty} n_{j} / j \leq 2
$$

each $f^{n_{j}}$ is injective on the component $B_{j}^{\prime}$ of $f^{-n_{j}}\left(B\left(f^{n_{j}}(x), \eta\right)\right)$ which contains $x$, has distortion bounded by 2 (i.e. $\left.\left(\forall y_{1}, y_{2} \in B_{j}^{\prime}\right)\left|\left(f^{n_{j}}\right)^{\prime}\left(y_{1}\right)\right| /\left|\left(f^{n_{j}}\right)^{\prime}\left(y_{2}\right)\right| \leq 2\right)$ and

$$
\left|\left(f^{n_{j}}\right)^{\prime}(x)\right| \geq \exp \left(n_{j} \chi_{\mu}(f) / 2\right) .
$$

For every (except at most two) $z$ there exists $t>0$ depending only on $\eta$ and $z$ such that for every $w \in J$ we have $f^{t}(B(w, \eta)) \ni z$. So for every $x$ as above we find $x_{j} \in B_{j}^{\prime}$ such that $f^{n_{j}+t}\left(x_{j}\right)=z$ and $\left|\left(f^{n_{j}}\right)^{\prime}\left(x_{j}\right)\right|^{-1} \geq \operatorname{dist}\left(x, x_{j}\right) / 2 \eta$. So $\left|\left(f^{n_{j}+t}\right)^{\prime}\left(x_{j}\right)\right|^{-1} \geq \operatorname{dist}\left(x, x_{j}\right) /\left(2 \eta \sup \left|\left(f^{t}\right)^{\prime}\right|\right)$, i.e. condition (ii) holds. Condition (i) also holds, with, say, $1+K^{-1}=\exp (\chi / 2)$. We conclude that $\mu$-a.e. $x$ belongs to $\Lambda_{c}(f)$, which by the definition of $\mathrm{HD}_{\text {mhyp }}$ proves the theorem.

(We have not made use here the property $\lim \sup _{j \rightarrow \infty} n_{j} / j \leq 2$. Taking this into account would suggest another definition of $\Lambda_{\mathrm{c}}$ and $\Lambda_{\mathrm{cw}}$.)

The proof above and the definition (0.2) justify the following definition.

Definition 2.3. $x \in \overline{\mathbb{C}}$ is called regular if it satisfies the property (2.1) with a number $\chi>0$ (we need not link $\chi$ to any measure). We denote the set of regular points by $\Lambda_{\text {reg }}(f)$.

We immediately obtain $\Lambda_{\text {reg }}(f) \subset \Lambda_{\mathrm{c}}(f)$ and $\operatorname{HD}_{\text {hyp }}(J) \leq \operatorname{HD}\left(\Lambda_{\text {reg }}(f)\right)$.

(Observe that if the property $\lim \sup n_{j} / j \leq 2$ is omitted in (2.1), the above inclusion still holds. We need this property later, to obtain $\Lambda_{\text {reg }}(f) \subset \Lambda_{\mathrm{tc}}(f)$.)

Recall that our aim is to prove

$$
\alpha(f)=\operatorname{HD}_{\text {mhyp }}(J)=\operatorname{HD}_{\text {hyp }}(J)=\operatorname{HD}\left(\Lambda_{\mathrm{c}}(f)\right)=\delta(f) .
$$

To this end in view of Theorems 2.1, 2.2 and (0.3) it only remains to prove $\delta(f) \leq \alpha(f)$, namely

Theorem 2.4. For every $\alpha$-conformal measure $\mu$ there exists $z$ such that $\delta(f, z) \leq$ $\alpha$.

Proof. Write $O_{n}($ Crit $):=\bigcup_{j=1}^{n}\left(\right.$ Crit). We have $\mu\left(O_{n}(\right.$ Crit $\left.)\right)=0$ (otherwise $\mu$ (Crit) $=\infty)$. Notice that for every $n \geq 0$

$$
\int_{J \backslash O_{n}(\mathrm{Crit})} P(z, \alpha, n) d \mu(z) \leq 1 .
$$

We obtain this by cutting a neighbourhood of $J$ into a finite number of topological discs of boundaries of measure $\mu$ equal to 0 containing $O_{n}$ (Crit). We consider all the branches of $f^{-n}$ on each such disc $U$. For each such branch $g$ we have, by the definition of conformal measure, $\mu(g(U))=\int_{U}\left|g^{\prime}\right|^{\alpha} d \mu$. Finally we sum these equalities over all the branches and $U$ 's. (Notice that we cannot assert the equality in (2.3) because of possible atoms of $\mu$ at critical points.)

For every $\varepsilon>0$ and $n$, by $(2.3)$

$$
\mu\{z: P(z, \alpha, n) \geq \exp n \varepsilon\} \leq \exp (-n \varepsilon) .
$$


So for $\mu$-a.e. $z$ we have $\lim \sup _{n \rightarrow \infty} \frac{1}{n} \log P(z, \alpha, n) \leq \varepsilon$. Hence there exists $z$ (even $\mu$-a.e.) such that $\lim \sup _{n \rightarrow \infty} \frac{1}{n} \log P(z, \alpha, n) \leq 0$. Hence $\delta(f) \leq \alpha$ by (1.1), the definition of $\delta(f)$.

Remark 2.5. The proof of (2.2) is over in the general case. However in the case where $f$ is a polynomial for example (or more generally if there exists a completely invariant basin of attraction to a sink) (for very non-polynomial examples see [P4]), one wants to consider $\delta(f, z)$ for $z$ in the basin, whereas the $z$ produced in the above proof belongs to $J$. We succeeded due to our ad hoc definition (1.1), but if we want to consider only $z$ in the basin, the main theorem in the next section becomes crucial.

Remark 2.6. The results of this section rely on the equality (0.3), namely on the quite sophisticated Denker-Urbański construction of a conformal measure with the exponent $\alpha=\operatorname{HD}_{\text {mhyp }}(J)$. Now, a posteriori, because $\delta(f, z)=\operatorname{HD}_{\text {mhyp }}(J)$, we obtain such a measure below, just by modifying slightly the Patterson-Sullivan construction (see also [DU1]). Unfortunately, without any additional assumptions on $f$ we do not know where this measure is supported. (We cannot, for example, exclude the possibility that it is supported by a circle in a Siegel disc $S$ and its pre-images for iterates of $f$ if $z \in S$.)

Fix $z$ and write $\delta=\delta(f, z)$. Assume $\delta<\infty$. For all $0<t \leq 1$ construct a sequence of positive numbers $\varphi_{t}(n), n=0,1,2, \ldots$, such that $\lim _{t \nearrow_{1}} \varphi_{t}(n)=\varphi_{1}(n)$ and

$$
\lim _{t \nearrow 1} \limsup _{n \rightarrow \infty} \sup _{t \leq \tau \leq 1} \max \left\{\varphi_{\tau}(n) / \varphi_{\tau}(n+1), \varphi_{\tau}(n+1) / \varphi_{\tau}(n)\right\}=1 ;
$$

moreover

$$
P(t):=\sum_{n} \varphi_{t}(n) P(z, \delta, n)<\infty \text { for } t<1 \quad \text { and } \quad \sum_{n} \varphi_{1}(n) P(z, \delta, n)=\infty .
$$

To find such sequences, proceed for example as follows. Write $a_{n}=P(z, \delta, n)$. Note that $\lim \sup _{n \rightarrow \infty} \frac{1}{n} \log a_{n}=0$ (see Appendix 2, Prop. A2.2). It is not difficult to find $b_{n}$ such that $b_{n} \geq a_{n}, \lim \sup _{n \rightarrow \infty} \frac{1}{n} \log b_{n}=0, b_{n} / b_{n+1} \rightarrow 1$ and there exists a sequence of integers $n_{i} \rightarrow \infty$ such that $b_{n_{i}}=a_{n_{i}}$ for every $i$. Next define

$$
\varphi_{t}(n)=b_{n}^{-1} P(z, \delta, n) \cdot t^{n} \text {. }
$$

For $t<1$ set

$$
\mu_{t}=\sum_{n} \sum_{f^{n}(y)=z} D_{y} \cdot \varphi_{t}(n)\left|\left(f^{n}\right)^{\prime}(y)\right|^{-\delta} / P(t),
$$

where $D_{y}$ is the Dirac delta measure at $y$. Finally define $\mu$ as a weak ${ }^{*}$ limit of $\mu_{t}$ as $t \nearrow 1$. As $P(1)=\infty$, the measure $\mu$ is $\delta$-conformal.

We end this section with the promised definition of $\Lambda_{\mathrm{tc}}$, the set of tree conical limit points. Recall first, [P3] or [PUZ], that all points of $\bigcup_{n>0} f^{-n}(z)$ can be organized in a geometric coding tree. Briefly: we define a graph $\overline{\mathcal{T}}$ by joining $z$ to its $f$-preimages by curves $\gamma^{1}, \ldots, \gamma^{d}$, and next consider all the curves $f^{-n}\left(\gamma_{j}\right)$. These curves are the edges of $\mathcal{T}$, whereas the points of $\bigcup_{n \geq 0} f^{-n}(z)$ are the vertices. Each sequence of symbols $\beta=\left(\beta_{0}, \beta_{1}, \ldots\right)$ for $1 \leq \beta_{n} \leq \bar{d}=\operatorname{deg} f$ corresponds to a line of an infinite sequence of edges and vertices in $\mathcal{T}$; we call this an infinite branch and denote it by $b(\beta)$. Now we are in the position to write 
Definition 2.7. $x$ is a tree conical limit point, i.e. $x \in \Lambda_{\mathrm{tc}}(f, z)$, iff there exist a branch $b(\beta)$ converging to $x$ and a sequence of vertices $x_{i} \in b(\beta)$ such that $x_{i}$ and integers $n_{i}$ satisfy (i), (ii) (from the definition of $\Lambda_{\mathrm{c}}$ ).

Now recall that the main theorem of [P3], Theorem B, implies that if

$$
\lim _{n \rightarrow \infty} \sup _{\gamma \in \mathcal{T}_{n}} \operatorname{diam} \gamma \rightarrow 0
$$

where $\mathcal{T}_{n}$ is the set of all the edges of the $n$-th generation (i.e. in $\left.f^{-n}\left(\bigcup_{j=1}^{d} \gamma^{j}\right)\right)$, then for every $\mu$ an $f$-invariant measure of positive Lyapunov exponent, $\mu$-a.e. $x$, is tree conical. (Formally $[\mathrm{P} 3, \mathrm{Th} . \mathrm{B}]$ gives only the accessibility along $b(\beta)$; verifying (i), (ii) in the proof requires making use of the positive Lyapunov exponent.) This concerns in fact all the points satisfying (2.1). Thus for $\Lambda_{\mathrm{tc}}(f)=\bigcap_{z} \Lambda_{\mathrm{tc}}(f, z)$ we have

$$
\Lambda_{\text {reg }}(f) \subset \Lambda_{\text {tc }}(f) .
$$

In the case when $f$ is a polynomial and $z \in A_{\infty}$ we can replace $b(\alpha)$ by an external ray $r$ and write $x_{i} \in r$ in Definition 2.7. This concerns simply connected $A_{\infty}$ as well as non-simply-connected; see [P3, Section 3].

\section{ON THE INDEPENDENCE OF $\delta$ AND $\Lambda$ FROM $z$}

We rely on the following combinatorial lemma.

Lemma 3.1. There exists $C>0$ such that for every set $W$ of $n \geq 0$ points in $\overline{\mathbb{C}}$ and $0<r<1 / 2$, for every $z_{1}, z_{2} \in \overline{\mathbb{C}} \backslash B(W, r)$ there exists a sequence of discs $B_{1}=B\left(q_{1}, \rho_{1}\right), \ldots, B_{k}=B\left(q_{k}, \rho_{k}\right)$ such that for every $j=1, \ldots, k$ each $2 B_{j}:=B\left(q_{j}, 2 \rho_{j}\right)$ is disjoint from $W, z_{1} \in B_{1}, z_{2} \in B_{k}, \bigcup_{j=1}^{k} B_{j}$ is connected and

$$
\begin{array}{cl}
k \leq C \sqrt{n} \sqrt{\log 1 / r} & \text { if } n \geq \log 1 / r, \\
k \leq C \log 1 / r & \text { if } n<\log 1 / r .
\end{array}
$$

Remark 3.2. Another formulation is to replace the number $k$ of discs by the number of squares in the Whitney covering [Stein] (our proof is in this spirit).

Notice that $k$ is often much larger than the distance $d_{h}\left(z_{1}, z_{2}\right)$ in the hyperbolic metric $d_{h}$ on $\overline{\mathbb{C}} \backslash W$ (suppose $\# W \geq 3$ ). If $z_{2}$ is fixed and the Euclidean distance $z_{1}$ to $W$ is $r$ very close to 0 , then $k$ is of order $\log 1 / r$ whereas $d_{h}\left(z_{1}, z_{2}\right)$ is of order $\log \log 1 / r$. One can replace $k$ by a comparable quantity: the quasi-hyperbolic distance between $z_{1}$ and $z_{2}$ in $\overline{\mathbb{C}} \backslash W$. See [Po]. $k$ is comparable also to infimum of hyperbolic distances in simply-connected domains in $\overline{\mathbb{C}} \backslash W$, see [H, Lemma 3]; however estimates in $[\mathrm{H}]$ without square roots are not sufficient for us.

Proof of Lemma 3.1. See Appendix 1.

Theorem 3.3 (Main Theorem). There exists $E \subset \overline{\mathbb{C}}$ of Hausdorff dimension 0 such that for every $z_{1}, z_{2} \in \overline{\mathbb{C}} \backslash E$ and every $\alpha \geq 0$ we have $\mathcal{P}(\alpha):=\mathcal{P}\left(z_{1}, \alpha\right)=$ $\mathcal{P}\left(z_{2}, \alpha\right)$ and $\delta:=\delta\left(f, z_{1}\right)=\delta\left(f, z_{2}\right)$. Moreover for every $z \in \overline{\mathbb{C}}$ we have $\mathcal{P}(\alpha) \leq$ $\mathcal{P}(z, \alpha)$ and $\delta \leq \delta(f, z)$.

Proof. For every $n \geq 1$ set $r_{n}=\exp (-\sqrt{n})$. Set $b_{n}=B\left(f^{n}(\right.$ Crit $\left.), r_{n}\right), E^{\prime}=$ $\bigcap_{N} \bigcup_{n>N} b_{n}$ and finally $E=E^{\prime} \cup O$ (Crit), where $O$ (Crit) $=\bigcup_{j=1}^{\infty} f^{j}$ (Crit). We have $\operatorname{HD}(E)=0$, because $\sum_{n=1}^{\infty} r_{n}^{\varepsilon}<\infty$ for every $\varepsilon>0$ and Crit is finite. Consider now arbitrary $z_{1}, z_{2} \in \overline{\mathbb{C}} \backslash E$. Then there exists $N$ such that for every $n>N, z_{\nu} \notin b_{n}$ 
for $\nu=1,2$. Let $a=\min _{\nu=1,2, j=1, \ldots, N} \operatorname{dist}\left(f^{j}(\right.$ Crit $\left.), z_{\nu}\right)$. Fix an arbitrary $n>N$ large enough that $r=r_{n}<a$. Set $W=\bigcup_{j=1}^{n} f^{j}$ (Crit). We apply now Lemma 3.1 and consider the discs $B_{1}, \ldots, B_{k}$.

We can assume that the diameters of the $B_{j}$ are smaller than a constant $\kappa$, depending only on $f$, so that for all the components of $f^{-n}\left(2 B_{j}\right)$ the diameters of the complements in $\overline{\mathbb{C}}$ are larger than a constant (for this it is sufficient to have $\kappa$ smaller than the minimal distance between two distinct points of a periodic orbit of period at least 3). This influences the constant $C$ in Lemma 3.1. We conclude that there exists a constant $\Delta>0$ (not depending on $n$ ) such that the distortion (see the definition in (2.1)) of all the branches of $f^{-n}$ on each $B_{j}$ is bounded by $\Delta$. (This is a variant of Koebe's distortion theorem; see for example [P5].)

Thus, for every $\alpha>0$, using Lemma 3.1 in the case $n \geq \log 1 / r$, and the inequality $\sqrt{n} \geq \log 1 / r$, for $n$ large enough

$$
P\left(z_{1}, \alpha, n\right) / P\left(z_{2}, \alpha, n\right) \leq \Delta^{\alpha k} \leq \Delta^{\alpha C n^{3 / 4}} .
$$

Hence

$$
\limsup _{n \rightarrow \infty} \frac{1}{n} \log P\left(z_{1}, \alpha, n\right)=\limsup _{n \rightarrow \infty} \frac{1}{n} \log P\left(z_{2}, \alpha, n\right),
$$

and so $\mathcal{P}\left(z_{1}, \alpha\right)=\mathcal{P}\left(z_{2}, \alpha\right)$ and $\delta\left(z_{1}\right)=\delta\left(z_{2}\right)$. The first part of the proof is over.

Consider now an arbitrary $z \in \overline{\mathbb{C}} \backslash O$ (Crit). The following holds, see [P1, Lemma 3]: $\forall\left(0<\varepsilon_{1}<1\right) \quad \exists\left(C>0, \varepsilon_{2}>0\right) \quad \forall\left(0<\varepsilon \leq \varepsilon_{2}\right) \quad \forall(m>0)$ the set $f^{-m}(z)$ contains at least the number $C(\operatorname{deg} f)^{\varepsilon_{1} m}$ of $(m, \varepsilon)$-separated points. (Recall that $x, y$ are called $(m, \varepsilon)$-separated if $\max _{j=0, \ldots, m} \operatorname{dist}\left(f^{j}(x), f^{j}(y)\right) \geq \varepsilon$.) Now fix $\varepsilon_{1}=$ $3 / 4$ and suppose that $m$ is small enough that $2 L^{m} r_{n} \leq \varepsilon_{2}$, where $L=\sup \left|f^{\prime}\right|$. To be concrete we set

$$
m=\log \left(\varepsilon_{2} / 2 r_{n}\right) / \log L=\log \left(\varepsilon_{2} / 2\right) / \log L+n^{1 / 2} / \log L .
$$

We calculate how large $m$ need be in order that $f^{-m}(\{z\}) \not \subset \bigcup_{j=1}^{n} B\left(f^{j}(\right.$ Crit $\left.), r_{n}\right)$. It is sufficient to have

$$
C(\operatorname{deg} f)^{3 m / 4}>n \#(\text { Crit }) .
$$

Indeed, if two distinct points $x, y \in f^{-m}(z)$ are in the same disc $B\left(f^{i}(c), r_{n}\right)$ for $c \in$ Crit, then their $f^{j}, j \leq m$, images are not more than $L^{m} 2 r_{n}$ apart, so $x$ and $y$ are not $\left(m, \varepsilon_{2}\right)$-separated.

Observe finally that $m$ defined in (3.1) satisfies (3.2) if $n$ is large enough.

So we have found $y \in f^{-m}(z) \backslash \bigcup_{j=1}^{n} B\left(f^{j}(\mathrm{Crit}), r_{n}\right)$. Fix $z_{2} \in \overline{\mathbb{C}} \backslash E$. For an arbitrary $\varepsilon>0$, if $n$ is large enough, $P(y, \alpha, n) \geq(\exp -n \varepsilon) P\left(z_{2}, \alpha, n\right)$ by the first part of the proof. So

$$
P(z, \alpha, n+m) \geq L^{-m}(\exp (-n \varepsilon)) P\left(z_{2}, \alpha, n\right) \geq(\exp (-2 n \varepsilon)) P\left(z_{2}, \alpha, n\right) .
$$

Again using (3.1) namely the fact that $m$ grows much slower than $n$, we obtain $\mathcal{P}\left(z_{2}, \alpha\right) \leq \mathcal{P}(z, \alpha)$ and $\delta(z) \geq \delta\left(z_{2}\right)$. The theorem is proved.

Theorem 3.4. There exists $E \subset \overline{\mathbb{C}}$ of Hausdorff dimension 0 such that for every $z_{1}, z_{2} \in \overline{\mathbb{C}} \backslash E$

$$
\Lambda_{\mathrm{cw}}(f):=\Lambda_{\mathrm{cw}}\left(f, z_{1}\right)=\Lambda_{\mathrm{cw}}\left(f, z_{2}\right)
$$

and for every $z \in \overline{\mathbb{C}}$ which has a backward trajectory omitting Crit, $\Lambda_{\mathrm{cw}}(f, z) \supset$ $\Lambda_{\mathrm{cw}}(f)$. 
Proof. We set $E$ the same as in the proof of Theorem 3.3. Let $x \in \Lambda_{\mathrm{cw}}\left(f, z_{1}, K\right)$, and let $x_{i}$ be a sequence of $f^{n_{i}}$-preimages of $z_{1}$ converging to $x$ satisfying (i) and (iii) in the definition of $\Lambda_{\mathrm{cw}}$. For each $n$ large enough there is a 1-to- 1 correspondence between $f^{-n}\left(z_{1}\right)$ and $f^{-n}\left(z_{2}\right)$. Namely, each branch of $f^{-n}$ in a neighbourhood of $z_{1}$ extends along the chain $B_{1}, \ldots, B_{k}$ (see the proof of Theorem 3.1) to $z_{2}$. So let $x_{i}$ correspond to $x_{i}^{\prime} \in f^{-n_{i}}\left(z_{2}\right)$. We obtain, for $n_{i}$ large enough,

$$
\frac{\left|\left(f^{n_{i}}\right)^{\prime}\left(x_{i}^{\prime}\right)\right|}{\left|\left(f^{n_{i}}\right)^{\prime}\left(x_{i}\right)\right|} \geq\left(1-(2 K)^{-1}\right)^{n_{i}}
$$

hence

$$
\left|\left(f^{n_{i}}\right)^{\prime}\left(x_{i}^{\prime}\right)\right| \geq\left(1+(3 K)^{-1}\right)^{n_{i}}
$$

if $K$ is large enough. We also find that the growth of $\operatorname{dist}\left(x_{i}, x_{i}^{\prime}\right)\left|\left(f^{n_{i}}\right)^{\prime}\left(x_{i}^{\prime}\right)\right|$ is slower than every exponential as $n_{i} \rightarrow \infty$. So

$$
\begin{aligned}
& \limsup _{i \rightarrow \infty} \log \left(\operatorname{dist}\left(x, x_{i}^{\prime}\right) \cdot\left|\left(f^{n_{i}}\right)^{\prime}\left(x_{i}^{\prime}\right)\right|\right) \\
\leq & \limsup _{i \rightarrow \infty} \log \left(\operatorname{dist}\left(x, x_{i}\right) \cdot\left|\left(f^{n_{i}}\right)^{\prime}\left(x_{i}^{\prime}\right)\right|\right) \leq 0 .
\end{aligned}
$$

So $x \in \Lambda_{\mathrm{cw}}\left(f, z_{2}, 3 K\right)$.

For an arbitrary $z$ which has a backward trajectory $\tau$ omitting Crit we find $z_{1} \in \tau$ such that $z_{1} \notin O$ (Crit), and next for every $n$ we find $y \in f^{-m}\left(z_{1}\right)$ as in the proof of Theorem 3.3 (i.e. not too close to $\bigcup_{j=1}^{n} f^{j}$ (Crit)). We can have additionally $\left|\left(f^{m}\right)^{\prime}(y)\right| \geq 1$, because most of the points in $f^{-m}\left(z_{1}\right)$ satisfy this - see for example [FLM]. So we can repeat the above estimates, using $m / n \rightarrow 0$.

\section{Appendix 1. Proof of Lemma 3.1.}

We can assume $z_{1}=-1, z_{2}=1$ in $\mathbb{C}$. Next we change the coordinates on the union of the triangles $\Delta_{1}$ with the vertices $-1, i,-i$ and $\Delta_{2}$ with the vertices $1, i,-i$ by a map $\Phi$ to the strip $T:=\{0 \leq \Im(z) \leq 1\}$ as follows: First deform $\Delta_{1}$ to $\Delta_{1}^{\prime}$ the domain between the straight rays from -1 through $i$ and $-i$, and the arc (containing 0 ) of the circle with origin at -1 and radius 1 . Next map $\Delta_{1}^{\prime}$ to $T \cap\{\Re z \leq 0\}$ by $z \mapsto \frac{i}{2}+\frac{2}{\pi} \log (z+1)$. On $\Delta_{2}$ write $\Phi=S \circ \Phi \circ S^{-1}$, where $S$ is the symmetry with respect to the imaginary axis.

The proof of the lemma now reduces to a construction of a "chain of squares" in $T$, i.e. a family of squares with a smallest possible number of elements, not intersecting $\Phi(W)$, whose union joins the interval $\Re z=-m$ to $\Re z=m$ for $m=$ $2 \log 1 / r$, with every two adjacent squares in the chain of a comparable size. We shall use certain triadic squares; see below.

We can assume $2 m=3^{l}, n=3^{t}$ for certain non-negative even integers $l, t$. Write $W^{\prime}=\Phi(W)$. We can adjust our construction by changing $\Phi$ slightly so that no $\Re z, \Im z$ for $z \in W^{\prime}$ is rational triadic.

Define inductively a sequence of horizontal strips

$$
T_{j}=\left\{z: a_{j} \cdot 3^{-j} \leq \Im z \leq\left(a_{j}+1\right) \cdot 3^{-j}\right\},
$$

where $T_{0}=T$, such that $T_{j+1} \subset T_{j}$ and $\#\left(W^{\prime} \cap T_{j}\right) \leq 3^{t-j}$ for every $j$. In particular, $W^{\prime} \cap T_{t+1}=\emptyset$.

Call every interval in $[-m, m]$ of the form $\left[b \cdot 3^{-v},(b+1) \cdot 3^{-v}\right]$, for an integer $b$ and a non-negative integer $v$, triadic or $v$-triadic. For every $v$-triadic $I$ denote by $K(I)$ the square $I \times T_{v}$. These are the squares we shall use to construct our chain. 
For every $v$-triadic $I$ we define

$$
\varphi(I):=\inf \left\{j \geq 0: W^{\prime} \cap T_{j} \cap\{\Re z \in I\}=\emptyset\right\} .
$$

We now define by induction certain families of triadic intervals. Let $\mathcal{I}_{0}$ consists of three $(t+1)$-triadic intervals with maximal possible $\varphi$.

Suppose that a family $\mathcal{I}_{j}$ is already defined and it consists of $(t+1-j)$-triadic intervals. Suppose $\# \mathcal{I}_{j}=3^{j+1}$. Suppose also that $j<t+1$ and $2 j-t<l$. The first inequality means that the $K(I)$ 's for $I \in \mathcal{I}_{j}$ are not yet of side 1 (the maximal possible size). The latter means that $3^{-(t+1-j)} 3^{j+1}<3^{l}$, i.e. $\mathcal{I}_{j}$ does not yet cover the whole segment $[-m, m]$.

We construct $\mathcal{I}_{j+1}$.

Every $I \in \mathcal{I}_{j}$ is contained in a $(t-j)$-triadic $\hat{I}$. We denote the set of such intervals $\hat{I}$ by $\mathcal{I}_{j+1}^{1}$ and include it in $\mathcal{I}_{j+1}$. If $I$ is not adjacent to $-m$ or $m$ and is not a middle interval of $\hat{I}$, we also include in $\mathcal{I}_{j+1}$ the $(t-j)$-triadic interval adjacent to $I$. We have up to now in $\mathcal{I}_{j+1}$ a family $\mathcal{I}_{j+1}^{2}$ of at most $2 \cdot 3^{j+1}$ elements. Complete it to $\mathcal{I}_{j+1}$ so that $\# \mathcal{I}_{j+1}=3^{j+2}$ by $(t-j)$-triadic intervals with maximal possible values of $\varphi$.

For every $j$ write $\mathcal{I}_{j}^{\text {ad }}$ for the family of all the $(t+1-j)$-triadic intervals in $\bigcup\left\{\hat{I}: I \in \mathcal{I}_{j}\right\} \backslash \bigcup\left\{I \in \mathcal{I}_{j}\right\}$

We include in our chain of squares joining $\Re z=-m$ to $\Re z=m$ all the squares $K(I)$ for

$$
I \in \mathcal{I}:=\mathcal{I}_{0} \cup \bigcup_{j} \mathcal{I}_{j-1}^{\mathrm{ad}} \cup \bigcup_{j}\left(\mathcal{I}_{j} \backslash \mathcal{I}_{j}^{1}\right) .
$$

The unions are over $j=1, \ldots,(l+t) / 2$ if $(l+t) / 2 \leq t+1$, i.e. $l \leq t+2$, in particular $\log 1 / r<n$. In this case $\mathcal{I}$ covers $[-m, m]$. The number of squares in the chain is bounded by $\sum_{j=0}^{(l+t) / 2} 3^{j+2} \leq$ Const $\sqrt{m} \sqrt{n}$, as asserted in the lemma.

In the case $l>t+2$ the union is over $j=1, \ldots, t+1$, and $[-m, m]$ is not yet covered by the intervals in $\mathcal{I}$. Fortunately all the points of $W^{\prime}$ lie over $I \in \mathcal{I}$, so we just add to the chain an appropriate family of unit squares (0-triadic). The total number of squares in the chain does not exceed $2 m+$ Const $n \geq$ Const $\log 1 / r$, as asserted in the lemma.

We end the proof by checking that all our squares are indeed disjoint from $W^{\prime}$. By induction we prove that for every $j$ and every interval $I$ with interior not intersecting $\bigcup \mathcal{I}_{j}$ we have $\varphi(I) \leq t-j$. In particular, for $I \in \mathcal{I}_{j+1} \backslash \mathcal{I}_{j+1}^{1}$ we have $K(I) \cap W^{\prime}=\emptyset$. This will end the proof, by the definition of $\mathcal{I}$.

For $j=0$, outside $\bigcup\left\{I \in \mathcal{I}_{0}\right\}$ we have $\varphi \leq t-1$, which is even better than demanded. So the same estimate is sufficient outside $\bigcup\left\{I \in \mathcal{I}_{1}\right\}$. To build $\mathcal{I}_{2}$ we added to $\mathcal{I}_{2}^{2}$ at least nine $(t-1)$-triadic intervals, so we have exhausted all for which $\varphi>t-2$.

In general, to build $\mathcal{I}_{j}$ we added to $\mathcal{I}_{j}^{2}$ at least $3^{j}(t+1-j)$-triadic intervals, so among them all for which $\varphi>t-j$.

\section{Appendix 2. Pressure.}

Proposition A2.1. For all $\alpha \geq 0$,

$$
\mathcal{P}(\alpha) \geq \log \operatorname{deg} f-\alpha \log \sup \left|f^{\prime}\right| .
$$


Proposition A2.2. $\mathcal{P}(z, \alpha)$ and $\mathcal{P}(\alpha)$ are Lipschitz continuous functions of $\alpha \geq 0$.

Proof. If there exists $\beta>0$ such that $\mathcal{P}(z, \beta)<\infty$, then for every $\varepsilon_{1}>0$ one has $P(z, \beta, n) \leq \exp n\left(\mathcal{P}(z, \beta)+\varepsilon_{1}\right)$ for $n$ large enough. Hence for every $y \in f^{-n}(z)$ one has $\left|\left(f^{n}\right)^{\prime}(y)\right|^{-\beta} \leq \exp n\left(\mathcal{P}(z, \alpha)+\varepsilon_{1}\right)$, and so $\left|\left(f^{n}\right)^{\prime}(y)\right| \geq \exp -n\left(\mathcal{P}(z, \beta)+\varepsilon_{1}\right) / \beta$. One has also $\left|\left(f^{n}\right)^{\prime}(y)\right| \leq \sup \left|f^{\prime}\right|^{n}$. So for every $\alpha \geq 0, \varepsilon$ such that $\alpha+\varepsilon \geq 0$ and $n$ large and $|\varepsilon| \leq \varepsilon_{0}$ we have

$$
\begin{aligned}
& P(z, \alpha, n) \exp \left(-|\varepsilon| n D\left(\varepsilon_{1}, z, \beta\right)\right) \leq P(z, \alpha+\varepsilon, n) \\
& \quad \leq P(z, \alpha, n)\left(\exp \left(|\varepsilon| n D\left(\varepsilon_{1}, z, \beta\right)\right)\right),
\end{aligned}
$$

where $D\left(\varepsilon_{1}, z, \beta\right):=\max \left\{\left(\mathcal{P}(z, \beta)+\varepsilon_{1}\right) / \beta, \log \sup \left|f^{\prime}\right|\right\}$. We conclude that

$$
|\mathcal{P}(z, \alpha+\varepsilon)-\mathcal{P}(z, \alpha)| \leq|\varepsilon| D(z)
$$

where $D(z):=\inf \{D(0, z, \beta): \mathcal{P}(z, \beta)<\infty\}$, and

$$
|\mathcal{P}(\alpha+\varepsilon)-\mathcal{P}(\alpha)| \leq D\left|\varepsilon_{0}\right| D(\beta),
$$

where $D=\inf _{z} D(z)$. Thus we have proved Lipschitz continuity $\forall \alpha \geq 0$.

We have proved also that either $(\forall \alpha>0) \mathcal{P}(z, \alpha)=\infty$ or $(\forall \alpha>0) \mathcal{P}(z, \alpha)$ $<\infty$.

Proposition A2.3. $\mathcal{P}(\alpha)$ is monotone decreasing.

Proof. For $z \notin E$ (see the proof of Th.3.3), for every $n$ large enough, all the branches of $f^{-n}$ exist on $B\left(z, r_{n}\right)$. Hence, by $r_{n}=\exp (-\sqrt{n})$ and by Koebe's distortion theorem, there exists $C>0$ such that for every $y \in f^{-n}(z)$ one has

$$
\left|\left(f^{n}\right)^{\prime}(y)\right|^{-1} \leq C \operatorname{diamComp}_{y} f^{-n}\left(B\left(z, r_{n} / 2\right) / r_{n} \leq C \exp \sqrt{n} .\right.
$$

Therefore for all $\alpha \geq 0$ and $\varepsilon \geq 0$ one has

$$
P(z, \alpha+\varepsilon, n) \leq P(z, \alpha, n) \sup _{f^{n}(y)=z}\left|\left(f^{n}\right)^{\prime}(y)\right|^{-\varepsilon} \leq P(z, \alpha, n) C^{\alpha} \exp \varepsilon \sqrt{n}
$$

and applying $\lim \sup _{n \rightarrow \infty} \frac{1}{n} \log$ to these expressions one gets

$$
\mathcal{P}(z, \alpha+\varepsilon) \leq \mathcal{P}(z, \alpha) .
$$

Recall now the definition of pressure by Denker and Urbański [DU2]:

Definition A2.4. Let $V$ be an open set in $J$ such that $V \supset$ Crit $\cap J$. Define $K(V):=J \backslash \bigcup_{n \geq 0} f^{-n}(V)$. As $K(V) \cap$ Crit $=\emptyset$, we can consider the standard topological pressure $\mathcal{P}\left(\left.f\right|_{K(V)},-\alpha \log \left|f^{\prime}\right|\right)$ for the map $\left.f\right|_{K(V)}$ and the real continuous function $-\alpha \log \left|f^{\prime}\right|$ on the compact set $K(V)$ (see [W]). Define

$$
\mathcal{P}_{\mathrm{DU}}(\alpha)=\sup _{V} \mathcal{P}\left(\left.f\right|_{K(V)},-\alpha \log \left|f^{\prime}\right|\right),
$$

where the supremum is taken over all $V$ considered above.

Two other definitions are of interest:

Definition A2.5. Hyperbolic pressure:

$$
\mathcal{P}_{\text {hyp }}(\alpha):=\sup _{X} \mathcal{P}\left(\left.f\right|_{X},-\alpha \log \left|f^{\prime}\right|\right),
$$

where the supremum is taken over all $f$-invariant isolated hyperbolic subsets of $J$. 
Definition A2.6. Hyperbolic variational pressure

$$
\mathcal{P}_{\text {hypvar }}(\alpha):=\sup _{\mu}\left\{\mathrm{h}_{\mu}(f)-\alpha \int \log \left|f^{\prime}\right| d \mu\right\},
$$

where the supremum is taken over all ergodic $f$-invariant measures of positive Lyapunov exponent, i.e. $\chi_{\mu}(f)=\int \log \left|f^{\prime}\right| d \mu>0$.

Definition A2.7. Variational pressure:

$$
\mathcal{P}_{\text {var }}(\alpha):=\sup _{\mu}\left\{\mathrm{h}_{\mu}(f)-\alpha \int \log \left|f^{\prime}\right| d \mu\right\},
$$

where the supremum is taken over all ergodic $f$-invariant measures on $J$.

Definition A2.8. Minimal conformal eigenvalue:

$$
\lambda(\alpha)=\inf \left\{\lambda>0: \exists \mu \text { a probability measure on } J \text { with Jacobian } \lambda\left|f^{\prime}\right|^{\alpha}\right\} .
$$

Here we say $\varphi: J \rightarrow \mathbb{R}, \varphi \geq 0$, is Jacobian for $\mu$ and $\left.f\right|_{J}$ if $\varphi$ is $\mu$-integrable and for every Borel set $E \in J$ on which $f$ is injective $\mu(f(E))=\int_{E} \varphi d \mu$. We write $\varphi=\operatorname{Jac}_{\mu}\left(\left.f\right|_{J}\right)$. (Compare the definition of $\alpha$-conformal at the end of the Introduction; there the Jacobian was equal to $\left|f^{\prime}\right|^{\alpha}$.)

Theorem A2.9. For every $0 \leq \alpha \leq \delta(f)$

$$
\log \lambda(\alpha)=\mathcal{P}(\alpha)=\mathcal{P}_{\text {var }}(\alpha)=\mathcal{P}_{\text {hypvar }}(\alpha)=\mathcal{P}_{\text {hyp }}(\alpha)=\mathcal{P}_{\mathrm{DU}}(\alpha) .
$$

All these functions are Lipschitz continuous and monotone decreasing on $\alpha \geq 0$.

Sketch of Proof. (It virtually repeats [DU2] and Section 2.)

1. We prove $(\forall \alpha \geq 0) \mathcal{P}(\alpha) \geq \mathcal{P}_{\text {hypvar }}(\alpha)$. For every $\mu$ as in the definition of $\mathcal{P}_{\text {hypvar }}$, for every $\varepsilon>0$ arbitrarily small and $n$ large enough, one constructs an $(n, \varepsilon)$-separated set $S_{n}$ such that

$$
\sum_{y \in S_{n}}\left|\left(f^{n}\right)^{\prime}(y)\right|^{-\alpha} \geq \exp n\left(h_{\mu}(f)-\alpha \int \log \left|f^{\prime}\right| d \mu-\varepsilon\right) .
$$

(This is Katok's construction; see for example [PUbook].) One can assume also that 2.1 holds (for a sequence of $n$ 's) and replace $y \in S_{n}$ by $y \in f^{-n-t}(z)$ as in proof of Theorem 2.2.

2. $\forall \alpha \geq 0 \mathcal{P}_{\text {hypvar }}(\alpha)=\mathcal{P}_{\text {hyp }}(\alpha)$. The $\geq$ inequality follows from the variational principle, see $[\mathrm{W}]$, and the obvious fact that every $f$-invariant probability measure on a hyperbolic set $X$ has positive Lyapunov exponent. The opposite inequality results from Katok's construction (the sets $S_{n}$ above are in fact constructed in hyperbolic sets).

3. If $\mathcal{P}_{\mathrm{DU}}(\alpha)>0$ then $\mathcal{P}_{\text {hypvar }}(\alpha) \geq \mathcal{P}_{\mathrm{DU}}(\alpha)$. For every $\varepsilon>0$ one can find, by the variational principle, an ergodic $f$-invariant $\mu$ on $K(V)$ such that $h_{\mu}(f)-$ $\alpha \int \log \left|f^{\prime}\right| d \mu>P\left(\left.f\right|_{K(V)},-\alpha \log \left|f^{\prime}\right|\right)-\varepsilon$. For $\varepsilon$ small enough the latter expression is positive, and $\int \log \left|f^{\prime}\right| d \mu \geq 0$ by [P2] or [DU2, Cor. 4.2]. Hence $h_{\mu}(f)$ and therefore $\int \log \left|f^{\prime}\right| d \mu$ are strictly positive; see [R] or [PUbook, Ch.8.1].

4. If $\mathcal{P}_{\text {var }}(\alpha)>0$, then $\mathcal{P}_{\text {var }}(\alpha)=\mathcal{P}_{\text {hypvar }}(\alpha)$ : Indeed, as in $\mathbf{3}$, if $h_{\mu}(f)-$ $\alpha \int \log \left|f^{\prime}\right| d \mu>\mathcal{P}_{\text {var }}(\alpha)-\varepsilon$ then for $\varepsilon$ small enough this is positive. As $\int \log \left|f^{\prime}\right| d \mu \geq$ 0 , by [P2], we obtain $h_{\mu}(f)$ and therefore $\int \log \left|f^{\prime}\right| d \mu$ strictly positive. Hence $\mathcal{P}_{\text {hypvar }}(\alpha) \geq \mathcal{P}_{\text {var }}(\alpha)-\varepsilon$ for every $\varepsilon>0$. 
5. We prove that for every $0 \leq \alpha<\delta(f)$ there exists a sequence of decreasing $V_{n}$ 's such that $\mathcal{P}\left(\left.f\right|_{K\left(V_{n}\right)},-\alpha \log \left|f^{\prime}\right|\right)>0$ and $\lim _{n \rightarrow \infty} \mathcal{P}\left(\left.f\right|_{K\left(V_{n}\right)},-\alpha \log \left|f^{\prime}\right|\right) \geq$ $\log \lambda(\alpha)$. In particular, $\mathcal{P}_{\mathrm{DU}}(\alpha)>0$ and $\mathcal{P}_{\mathrm{DU}}(\alpha) \geq \log \lambda(\alpha)$.

Take $V_{n}$ of the form $\bigcup_{j \geq 0} f^{-j}\left(\hat{V}_{n}\right)$, where $\hat{V}_{n}$ is a union of small discs $B\left(x_{c}, r_{n}\right)$ for a distinguished point $x_{c}$ in the $\omega$-limit set for each $c \in$ Crit $\cap J$. One can choose $x_{c}$ so that there exists $C>0$ such that for a sequence $j_{k} \rightarrow \infty$ we have $\left|\left(f^{j_{k}}\right)^{\prime}\left(x_{c}\right)\right| \geq C$, [P2]. Hence [DU2, Lemma 5.4] for $r_{n} \searrow 0$ there exists a sequence of measures $\mu_{n}$ on $K\left(V_{n}\right)$ with Jacobians

$$
\operatorname{Jac}_{\mu_{n}}\left(\left.f\right|_{K\left(V_{n}\right)}\right)=\lambda_{n}\left|f^{\prime}\right|^{\alpha}
$$

for $1 \leq \lambda_{n} \leq \exp \mathcal{P}\left(\left.f\right|_{K\left(V_{n}\right)},-\alpha \log \left|f^{\prime}\right|\right)$ on $K\left(V_{n}\right) \backslash \partial \hat{V}_{n}$ Here we mean that $\left|f^{\prime}\right|^{\alpha}$ is $\mu_{n}$-integrable on $K(V)$ such that for every Borel $E \subset K\left(V_{n}\right) \backslash \partial \widehat{V}_{n}$ on which $f$ is injective, $\mu(f(E))=\int_{E} \lambda_{n}\left|f^{\prime}\right|^{\alpha} d \mu$. Moreover for every $E \in \partial \hat{V}_{n}$ on which $f$ is injective we have

$$
\mu_{n}(f(E)) \geq \lambda_{n} \int_{E}\left|f^{\prime}\right|^{\alpha} d \mu_{n} .
$$

A weak* limit $\mu=\lim _{n_{k} \rightarrow \infty} \mu_{n_{k}}$ has Jacobian in the sense of Definition A2.8 satisfying

$$
\operatorname{Jac}_{\mu}\left(\left.f\right|_{J}\right)=\lambda\left|f^{\prime}\right|^{\alpha}
$$

for $\lambda=\lim \lambda_{n_{k}}$. We have made use here of the fact that $\mu$ has no atoms at $f\left(x_{c}\right)$, because liminf $\lambda_{n} \geq 1$ and $\sum_{j}\left|\left(f^{j}\right)^{\prime}\left(x_{c}\right)\right|^{\alpha}=\infty$. Such atoms would cause troubles with an estimate of the Jacobian (see [DU2]) because the $\partial \hat{V}_{n}$ accumulate at $x_{c}$ and we have only an inequality in (A2.2), and so only an inequality for $\mu$ for $E=\left\{x_{c}\right\}$.

We have applied here $\mathcal{P}\left(\left.f\right|_{K\left(V_{n}\right)},-\alpha \log \left|f^{\prime}\right|\right)>0$. If this were not true, we would find $\alpha_{n} \leq \alpha$ such that $1 / n>\mathcal{P}\left(\left.f\right|_{K\left(V_{n}\right)},-\alpha_{n} \log \left|f^{\prime}\right|\right)>0$, and the above construction would give a $\beta$-conformal measure with exponent $\beta \leq \alpha$. Then, however, by (2.2) (Th. 2.4), $\delta(f) \leq \beta \leq \alpha<\delta(f)$, contradiction.

We have also made use here of the inequality $\lambda<\infty$, true because the $\mathcal{P}\left(\left.f\right|_{K\left(V_{n}\right)},-\alpha \log \left|f^{\prime}\right|\right)$ were upper bounded by $\mathcal{P}(\alpha)$ due to the already proved 1-3.

6. $\forall \alpha \geq 0 \log \lambda(\alpha)=\mathcal{P}(\alpha)$. Indeed, if $\operatorname{Jac}_{\mu}\left(\left.f\right|_{J}\right)=\lambda\left|f^{\prime}\right|^{\alpha}$ then we find $z$ for which $\mathcal{P}(z, \alpha) \leq \log \lambda$ as in the proof of Theorem 2.4. Hence $\mathcal{P}(\alpha) \leq \log \lambda(\alpha)$. The opposite inequality follows already from $\mathbf{1}-\mathbf{5}$ for $\alpha<\delta(f)$. For an arbitrary $\alpha \geq 0$ one can construct $\mu$ with Jacobian $\exp \mathcal{P}(z, \alpha)$ by the Patterson-Sullivan method as in Remark 2.6.

7. The Lipschitz continuity and monotone decrease of $\mathcal{P}_{\text {var }}(\alpha)$, $\mathcal{P}_{\text {hypvar }}(\alpha)$, $\mathcal{P}_{\text {hyp }}(\alpha)$ and $\mathcal{P}_{\mathrm{DU}}(\alpha)$ follow from the definitions (variational principle) and from the fact that $0 \leq \int \log \left|f^{\prime}\right| d \mu \leq \sup \left|f^{\prime}\right|$ for every invariant probability measure $\mu$ on $J$ [P2]. Therefore (A2.1) extends from $\alpha<\delta$ to $\alpha=\delta$.

Corollary A2.10. $\mathcal{P}(\alpha)$ is a strictly decreasing, convex function on $0 \leq \alpha \leq \delta(f)$.

Proof. This is so for the affine function $h_{\mu}(f)-\alpha \int \log \left|f^{\prime}\right| d \mu$ for each $\mu$ of positive Lyapunov exponent, so the supremum over the $\mu$ 's, namely $\mathcal{P}_{\text {hypvar }}(\alpha)$, is monotone decreasing and convex. As this attains 0 at $\delta(f)$, the convexity implies it is strictly decreasing. One can proceed also directly. Proposition A2.3 gives monotonicity and Schwarz inequality gives convexity just from the definition of $\mathcal{P}(\alpha)$. 
Remark A2.11. The equalities (A2.1) immediately yield the equalities (2.2). Namely, the least zeros of $\log \lambda(\alpha), \mathcal{P}(\alpha), \mathcal{P}_{\text {hypvar }}(\alpha), \mathcal{P}_{\text {hyp }}(\alpha)$ are equal to $\alpha(f), \delta(f)$, $\operatorname{HD}_{\text {mhyp }}(J), \operatorname{HD}_{\text {hyp }}(J)$, respectively.

\section{Appendix 3. Some properties of $\Lambda_{\mathrm{c}}, \Lambda_{\mathrm{cw}}$ AND OTHER DEFINITIONS OF "CONICAL".}

After distributing the first version of this paper I was asked about a relation between the definitions of "conical limit set" in the recent preprints [LM], [U2], [DMNU] and my definitions. In [U2] and [DMNU] $x$ is called conical if there exists $\eta>0$ and a sequence of integers $n_{j} \rightarrow \infty$ such that each $f^{n_{j}}$ is injective on $\mathrm{Comp}_{x} f^{-n_{j}}\left(B\left(f^{n_{j}}(x), \eta\right)\right)\left(\mathrm{Comp}_{x}\right.$ means the component containing $\left.x\right)$. We denote the set of points "conical" in this sense by $\Lambda_{\mathrm{U}}(f)$.

Of course $\Lambda_{\mathrm{U}}(f) \supset \Lambda_{\text {reg }}(f)$.

Suppose that $f$ has no critical points in $J$ but the set $P$ of periodic parabolic points $\left(f^{k}(p)=p,\left(f^{k}\right)^{\prime}(p)\right.$ is a root of unity) is non-empty. Then $J=\Lambda_{\mathrm{U}}(f) \cup$ $\bigcup_{n \geq 0} f^{-n}(P)$ and $\Lambda_{\mathrm{U}}(f) \cap \bigcup_{n>0} f^{-n}(P)=\emptyset$. This is similar to the geometrically finite Kleinian groups case; see [Maskit, VI.C.3]. This was in fact a motivation for the definition of $\Lambda_{\mathrm{U}}(f)$ in [U2]. Unfortunately this is not so for $\Lambda_{\mathrm{cw}}$.

Proposition A3.1. For each $f$ with no critical points in $J$, when $P \neq \emptyset$ there exist points which are neither in $\bigcup_{n \geq 0} f^{-n}(P)$ nor in $\Lambda_{\mathrm{cw}}(f)$.

Proof. Consider a finite Markov partition of $J$, attribute the symbol 0 to all its cells whose closures contain a periodic parabolic point $p$, and attribute other symbols to other cells. For each $x \in J$ choose a sequence $a_{j}(x)$ of symbols so that each is attributed to a cell whose closure contains $f^{j}(x)$. We prove that if

$$
\lim _{n \rightarrow \infty} \#\left\{0 \leq j<n: a_{j}(x)=0\right\} / n=1,
$$

then $x \notin \Lambda_{\mathrm{cw}}(f, z)$.

Indeed, suppose $y=x_{i}$ satisfy (i), (iii) for $n=n_{i}$. Hence for a constant $0<A<$ 1 , for $n$ large enough, $\operatorname{dist}(x, y) \leq A^{n}$. We shall prove that in consequence

$$
\operatorname{dist}\left(f^{n}(x), f^{n}(y)\right) \leq B^{n} \operatorname{dist}(x, y),
$$

where $B \searrow 1$ as $n \rightarrow \infty$. Indeed, join $x$ to $y$ by an interval $I$. Fix an arbitrary $B$ with $A^{-1}>B>1$. By (a), since $\left|f^{\prime}\right| \approx 1$ near $P$, there exists $n(B)$ such that for every $t \geq n(B)$ we have $\left|\left(f^{t}\right)^{\prime}(x)\right| \leq(\sqrt{B})^{t}$ and for every $w \in J, v \in \overline{\mathbb{C}}$ with $\operatorname{dist}(w, v) \leq(A B)^{n(B)}$ we have $\left|f^{\prime}(v)\right| /\left|f^{\prime}(w)\right|<\sqrt{B}$. Suppose that (b) fails and $j$ (with $n(B) \leq j \leq n$ ) is the first integer such that there exists $z \in I$ satisfying $\operatorname{dist}\left(f^{j}(x), f^{j}(z)\right)>B^{j} \operatorname{dist}(x, z)$. In particular, for every $n(B) \leq j^{\prime}<j$ and $v \in[x, z]$ we have $\operatorname{dist}\left(f^{j^{\prime}}(x), f^{j^{\prime}}(v)\right] \leq B^{j^{\prime}} A^{n} \leq(A B)^{n} \leq(A B)^{n(B)}$. We also have $\operatorname{dist}\left(f^{j^{\prime}}(x), f^{j^{\prime}}(v)\right] \leq(A B)^{n(B)}$ for $0 \leq j^{\prime}<n(B)$ provided $A^{n}$ is small, i.e. $n$ is large enough. Hence

$$
\operatorname{dist}\left(f^{j}(x), f^{j}(z)\right) \leq \int_{[x, z]}\left|\left(f^{j}\right)^{\prime}(v)\right| d v \leq(\sqrt{B})^{j}(\sqrt{B})^{j} \operatorname{dist}(x, z)=B^{j} \operatorname{dist}(x, z),
$$

a contradiction. So (b) has been proved.

We conclude from (a) and (b) that $\left|\left(f^{n}\right)^{\prime}(y)\right| \leq \operatorname{Const} B^{n}$ with $B \searrow 1$, which contradicts (i). 
(I thank M. Urbański for a simplification.)

We conclude that $\Lambda_{\mathrm{U}}(f) \not \subset \Lambda_{\mathrm{cw}}(f)$.

Clearly the sets $\Lambda_{\mathrm{U}}(f), \Lambda_{\mathrm{c}}(f, z)$ and $\Lambda_{\mathrm{cw}}(f, z)$ are forward invariant.

For our $\Lambda$ 's also the backward invariance holds (unlike for $\Lambda_{U}$ - see for example the paragraph preceding Definition A3.5).

Proposition A3.2. For every rational $f$ and every non-exceptional $z$ (in the sense of Theorem 3.1), $f^{-1}\left(\Lambda_{\mathrm{c}}\right)=\Lambda_{c}$ and $f^{-1}\left(\Lambda_{\mathrm{cw}}\right)=\Lambda_{\mathrm{cw}}$.

Proof. Suppose $x=f\left(x^{\prime}\right) \in \Lambda_{\mathrm{c}}(f, z)$. Assume $x^{\prime} \in \operatorname{Crit}(f)$, as for $x^{\prime}$ non-critical $x^{\prime} \in \Lambda_{\mathrm{c}}(f, z)$ follows immediately. Let $x_{i}$ be points approximating $x$ according to Definition 1.2. Then, as $z \notin E$, there exists a branch of $f^{-n_{i}}$ on $B\left(z, \exp \left(-\sqrt{n_{i}}\right)\right)$ mapping $z$ to $x_{i}$, for $n_{i}$ large enough. So by bounded $\operatorname{distortion} \operatorname{dist}\left(x, x_{i}\right) \geq$ Const $\left|\left(f^{n_{i}}\right)^{\prime}\left(x_{i}\right)\right|^{-1} \exp \left(-\sqrt{n_{i}}\right)$. Hence, for $x_{i}^{\prime}$ the $f$-preimage of $x_{i}$, near $x^{\prime}$, we obtain

$$
\left|\left(f^{n_{i}+1}\right)^{\prime}\left(x_{i}^{\prime}\right)\right|^{-1} \leq \operatorname{Const}\left(1+K^{-1}\right)^{-(1 / \nu) n_{i}} \exp \left((1-1 / \nu) \sqrt{n_{i}}\right),
$$

so (i) holds for $x^{\prime}$ with $x_{i}^{\prime}, n_{i}+1$ for $i$ large enough (and new $K$ ). Here $\nu$ is the multiplicity of $f$ at $x^{\prime}$.

The property (ii) for $x^{\prime}$ follows immediately from

$$
\left|f^{\prime}\left(x_{i}^{\prime}\right)\right|^{-1} \geq \frac{\operatorname{dist}\left(x^{\prime}, x_{i}^{\prime}\right)}{2 \nu \operatorname{dist}\left(x, x_{i}\right)} .
$$

The proof for $\Lambda_{\mathrm{cw}}$ is similar.

The following rational maps are of interest (see $[\mathrm{PR}]$ ).

Definition A3.3. $f$ is called topological Collet-Eckmann if there exist $M, N, \eta>0$ such that for every $x \in J$

$$
\begin{gathered}
\exists n_{j} \rightarrow \infty, n_{j} \leq N j, \quad \text { such that each } f^{n_{j}} \text { has degree } \\
\text { at most } M \text { on } \operatorname{Comp}_{x} f^{-n_{j}} B\left(f^{n_{j}}(x), \eta\right) .
\end{gathered}
$$

(Recall that $\operatorname{Comp}_{x}$ means the component containing $x$.)

Proposition A3.4. For every rational $f$, every $x \in J(f)$, for which there exist $M, N, \eta>0$ such that (A3.1) is satisfied, is conical. More precisely, $x \in \Lambda_{\mathrm{c}}(f, z)$ for every $z \notin O$ (Crit). In particular, if $f$ is topological Collet-Eckmann, then $J(f)=\Lambda_{\mathrm{c}}(f)$.

Proof. It follows from the proof of [PR, Prop.3.1] that there exist $0<\xi<1, \eta>0$ and a sequence of integers $n_{j} \rightarrow \infty$ such that for each

$$
W_{j}:=\operatorname{Comp}_{x} f^{-n_{j}}\left(B\left(f^{n_{j}}(x), \eta\right)\right)
$$

we have together with (A3.1) also

$$
\operatorname{diam} W_{j} \leq \xi^{n_{j}} .
$$

Due to (A3.1) we can also assume, taking $\eta$ small enough, that all $f^{k}\left(W_{j}\right), k=$ $0,1, \ldots, n_{j}$, have small diameters. See $[\mathrm{M}]$.

(In [P3, Lemma 3.6], it has been proved that (A3.2) holds for all $n$.)

Fix an arbitrary $n=n_{j}$. We claim that there exists a disc $D \subset B\left(f^{n}(x), \eta\right)$ of diameter at least $A \eta$ for a constant $A$ depending only on $M$ such that there exists $y \in W \cap f^{-n}(D)$ satisfying

$$
\left|\left(f^{n}\right)^{\prime}(y)\right| \geq A \xi^{-n}
$$


and

$$
\operatorname{dist}(x, y) \leq A^{-1}\left|\left(f^{n}\right)^{\prime}(y)\right|^{-1} .
$$

Indeed, there exists an integer $0 \leq k \leq M$ such that the annulus

$$
B\left(f^{n}(x), \eta \frac{k+1}{M+1}\right) \backslash B\left(f^{n}(x), \eta \frac{k}{M+1}\right)
$$

does not contain any critical value for $\left.f^{n}\right|_{W}$. We choose $D$ an arbitrary disc in the annulus

$$
B\left(f^{n}(x), \eta \frac{3 k+2}{3(M+1)}\right) \backslash B\left(f^{n}(x), \eta \frac{3 k+1}{3(M+1)}\right) .
$$

Denote $W^{\prime}=\operatorname{Comp}_{x} f^{-n}\left(B\left(f^{n}(x), \eta(3 k+2) / 3(M+1)\right)\right)$ and fix $y \in W^{\prime} \cap f^{-n}(D)$. Let $0=m_{0}<m_{1}<m_{2}<\ldots<m_{M^{\prime}}=n$ be all consecutive integers such that $f^{m_{t}}\left(W^{\prime}\right)$ contains an $f$-critical point, except maybe $t=0$ and $t=M^{\prime}$. (Observe that $M^{\prime} \leq M(\operatorname{deg} f)+1$.) Then for every $m_{t}, t<M^{\prime}$, we have

$$
\left.\frac{\operatorname{diam} f^{m_{t}+1}\left(W^{\prime}\right)}{\operatorname{diam} f^{m_{t+1}}\left(W^{\prime}\right)} \approx \mid f^{m_{t+1}-m_{t}-1}\right)\left.^{\prime}\left(f^{m_{t}+1}(y)\right)\right|^{-1}
$$

and

$$
\frac{\operatorname{diam} f^{m_{t}}\left(W^{\prime}\right)}{\operatorname{diam} f^{m_{t}+1}\left(W^{\prime}\right)} \approx\left|f^{\prime}\left(f^{m_{t}}(y)\right)\right|^{-1} .
$$

Here $\approx$ means the ratios of the left and right sides, and vice versa, are bounded by a constant depending only on $M$. The former $\approx$ follows from bounded distortion; the latter holds because the distance of $f^{m_{t}+1}(y)$ from $f\left(\operatorname{Crit}(f) \cap f^{m_{t}}\left(W^{\prime}\right)\right)$ is at least $C_{t} \operatorname{diam} f^{m_{t}+1}\left(W^{\prime}\right)$ for a constant $C_{t}>0$.

Combining (A3.5) and (A3.6) over all $t$, we obtain for a constant $A>0$

$$
A \operatorname{diam} W^{\prime} \leq\left|\left(f^{n}\right)^{\prime}(y)\right|^{-1} \eta \leq A^{-1} \operatorname{diam} W^{\prime} .
$$

The right inequality together with (A3.2) gives (A3.3). The left inequality in (A3.7) immediately gives (A3.4).

(The above proof is only sketched. A precise proof needs induction over decreasing $t$ 's to control distortion, and constants $C_{t}$. For details see [P5, proof of L.1.4, (1.4)]; though only the left inequality in (A3.7) is proved there, the technique is the same.)

It is known that there exists $k$, depending only on $z$ and on the diameter of $D$ (i.e. on $\eta$ and $M$ ), such that $f^{k}(D) \ni z$. So for every $n_{j}$ we could choose $y$ with $f^{n_{j}+k}(y)=z$. Moreover, $\left|\left(f^{k}\right)^{\prime}\left(f^{n_{j}}(y)\right)\right|>$ Const $>0$ (because $z \notin O$ (Crit)). This and (A3.3) give (i) for $n_{j}+k$. The upper bound for $\left|\left(f^{k}\right)^{\prime}\right|$ and (A3.4) give (ii).

Notice that this proposition, (2.2) and [PR, L.2.2] imply for $f$ Collet-Eckmann that $H D_{\text {hyp }}(J)=H D(J)$.

(Thus we have obtained a new proof of a part of [P5, Th.A]. Recall that $f$ is called Collet-Eckmann if for every $c \in$ Crit $\cap J$, whose forward trajectory does not meet other critical points, $\left|\left(f^{n}\right)^{\prime}(f(c))\right|$ grows exponentially fast as $n \rightarrow \infty$. [PR, L.2.2] says that Collet-Eckmann implies topological Collet-Eckmann.)

Notice that, by Proposition A3.4, if $f$ is topological Collet-Eckmann with Crit $\cap$ $J \neq \emptyset$, then $\Lambda_{\mathrm{c}}(f) \not \subset \Lambda_{\mathrm{U}}(f)$.

This is so because, by the definition, $c \notin \Lambda_{\mathrm{U}}$.

Recall [CJY] that $f$ is called semi-hyperbolic if it has no recurrent critical points and no parabolic periodic points. This class of maps is contained in the class of 
topological Collet-Eckmann maps. It follows from [U1] that if $f$ is semi-hyperbolic, then all points in $J$ except $\bigcup_{n \geq 0} f^{-n}$ (Crit) belong to $\Lambda_{\mathrm{U}}(f)$. So $\Lambda_{\mathrm{U}}(f)$ is not backward invariant.

M. Lyubich and M. Minsky's conical limit set, defined in [LM], which we denote by $\Lambda_{\mathrm{LM}}$, has a complicated definition and we shall not rewrite it here. Instead, let us introduce the following notion:

Definition A3.5. We call $x \in J$ strong LM-conical if there exist $\eta, M>0$ and sequences of integers $n_{j} \rightarrow \infty, k_{i} \rightarrow \infty$ and $i(j) \rightarrow \infty$ such that for every $n_{j}$ and $i=1, \ldots, i(j)$ the map $f^{n_{j}-k_{i}}$ has degree bounded by $M$ on the set

$$
\operatorname{Comp}_{x} f^{-\left(n_{j}-k_{i}\right)}\left(B\left(f^{n_{j}-k_{i}}(x), \eta\right)\right) .
$$

Denote the set of all strong LM-conical points by $\Lambda_{\mathrm{sLM}}(f)$

One can show that $\Lambda_{\mathrm{sLM}}(f) \subset \Lambda_{\mathrm{LM}}(f)$. In [LM], $x$ together with a backward trajectory $\hat{x}$ with "parabolic global unstable leaf" is considered. Note that such a $\hat{x}$ always exists. Just take $\hat{x}$ coverging to a repelling periodic orbit.

By [LM, Prop. 8.8 and L.8.4], if $f$ is semi-hyperbolic, then $\Lambda_{\mathrm{LM}}(f)=J$. It is easy to see that if $x$ satisfies (A3.1) then $x$ is strong LM-conical. Thus we obtain $\Lambda_{\mathrm{LM}}(f)=J$ for every topological Collet-Eckmann map $f$.

Along the way we obtain also $\Lambda_{\mathrm{LM}}(f) \supset \Lambda_{\mathrm{sLM}}(f) \supset \Lambda_{\text {reg }}(f)$.

Notice finally that, similarly to $\Lambda_{\mathrm{cw}}(f)$, one has

$$
\operatorname{HD}\left(\Lambda_{\mathrm{U}}(f)\right)=\operatorname{HD}\left(\Lambda_{\mathrm{LM}}(f)\right)=\operatorname{HD}_{\mathrm{hyp}}(J)
$$

$\operatorname{HD}\left(\Lambda_{\mathrm{U}}(f)\right)=\operatorname{HD}_{\text {hyp }}(J)$ follows from $\operatorname{HD}\left(\Lambda_{\mathrm{U}}(f)\right) \leq \alpha$ for every $\alpha$-conformal measure; see [U2] and [DMNU] (and from (2.2): $\alpha(f) \leq \operatorname{HD}_{\text {hyp }}(J), \quad \Lambda_{\mathrm{U}}(f) \supset$ $\Lambda_{\text {reg }}(f)$ and $\left.\operatorname{HD}_{\text {hyp }}(J) \geq \operatorname{HD}\left(\Lambda_{\text {reg }}(f)\right)\right)$.

This follows, however, as well as the second equality in (A3.8), from Proposition A3.7 below.

Definition A3.6. We say that $x \in \Lambda_{\mathrm{LM} 1}(f)$ if there exist $\eta, M>0$ and a sequence of integers $n_{j} \rightarrow \infty$ such that each $f^{n_{j}}$ has degree bounded by $M$ on $\operatorname{Comp}_{x} f^{-n_{j}}\left(B\left(f^{n_{j}}(x), \eta\right)\right)$.

Of course $\Lambda_{\mathrm{LM} 1}(f) \supset \Lambda_{\mathrm{U}}(f)$, and by [LM, Prop.8.7] $\Lambda_{\mathrm{LM} 1}(f) \supset \Lambda_{\mathrm{LM}}(f)$.

Proposition A3.7. $\mathrm{HD}\left(\Lambda_{\mathrm{LM} 1}(f)\right) \leq \alpha(f)$. Hence $\operatorname{HD}\left(\Lambda_{\mathrm{LM} 1}(f)\right)=\operatorname{HD}_{\mathrm{hyp}}(J)$.

Proof. The inequality follows as in [P5, Proof of Th. A] from $\mu\left(B\left(x, r_{j}\right)\right) \geq \operatorname{Constr}_{j}^{\alpha}$ for $x \in \Lambda_{\mathrm{LM} 1}(f)$ for $\mu$ any $\alpha$-conformal measure and $r_{j}=r_{j}(x) \rightarrow 0$ as $j \rightarrow \infty$. Const depends on $M$ only. The latter inequality uses bounded distortion for finite criticality as [P5, L.1.4].

Question. Is every conformal measure $\mu$ on $\Lambda_{\mathrm{c}}(f)$ ergodic? Is $\mu$ unique, provided it has no atoms at critical points?

\section{ADDED IN PROOF}

Recently the same estimate as in our Lemma 3.1 with an estimate for $C$ was proved by R. R. Hall and W. K. Hayman in the preprint "Successive ordinates of the zeros of the Riemann zeta function". 


\section{REFERENCES}

[BJ] C. Bishop, P. Jones, Hausdorff dimension and Kleinian groups, Acta Math. 179 (1997), 1-39. CMP 98:05

[CJY] L. Carleson, P. Jones, J.-Ch. Yoccoz, Julia and John, Bol. Soc. Bras. Mat. 25 (1994), 1-30. MR 95d:30040

[DMNU] M. Denker, D. Mauldin, Z. Nitecki, M. Urbański, Conformal measures for rational functions revisited, Fund. Math. 157.2-3 (1998), 161-173. CMP 98:16

[DU1] M. Denker, M. Urbański, On the existence of conformal measures, Trans. AMS $\mathbf{3 2 8 . 2}$ (1991), 563-587. MR 92k:58155

[DU2] - On Sullivan's conformal measures for rational maps of the Riemann sphere, Nonlinearity 4 (1991), 365-384. MR 92f:58097

[FLM] A. Freire, A. Lopes, R. Mañé, An invariant measure for rational maps, Bol. Soc. Bras. Mat. 141 (1983), 45-62. MR 85m:58110b

$[\mathrm{H}] \quad$ W. K. Hayman, The maximum modulus and valency of functions meromorphic in the unit circle, Acta Math. 86 (1951), 89-257. MR 13:546a

[LM] M. Lyubich, Y. Minsky, Laminations in holomorphic dynamics, J. Differential Geometry 47 (1997), 17-94.CMP 98:07

[M] R. Mañé, On a theorem of Fatou, Bol. Soc. Bras. Mat. 24 (1993), 1-12. MR 94g:58188

[Maskit] B. Maskit, Kleinian Groups, Springer, 1988. MR 90a:30132

$[\mathrm{McM}] \quad$ C. T. McMullen, Renormalization and 3-Manifolds which Fiber over the Circle, Annals of Mathematical Studies 142, Princeton University Press, 1996. MR 97f:57022

[N] P. J. Nicholls, The Ergodic Theory of Discrete Groups, LMS Lect. Notes 143, Cambridge Univ. Press, 1989. MR 91i:58104

[Pa] S. J. Patterson, Lectures on measures on limit sets of Kleinian groups., Analytical and Geometric Aspects of Hyperbolic Space. (D. B. A. Epstein., eds.), LMS Lect. Notes 111, Cambridge Univ. Press, 1986, pp. 281-323. MR 89b:58122

[Po] Ch. Pommerenke, Boundary Behaviour of Conformal Maps, Springer, 1992. MR 95b:30008

[P1] F. Przytycki, On the Perron-Frobenius-Ruelle operator for rational maps on the Riemann sphere and for Hölder continuous functions, Bol. Soc. Bras. Mat. 20.2 (1990), 95-125. MR 93b:58120

[P2] Lyapunov characteristic exponents are nonnegative, Proc. AMS 119.1 (1993), 309-317. MR 93k:58193

[P3] _ Accessibility of typical points for invariant measurs of positive Lyapunov exponents for iteration of holomorphic maps, Fund. Math. 144 (1994), 259-278. MR 95g:58190

[P4] _ Iterations of rational functions: which hyperbolic components contain polynomials?, Fund. Math. 149 (1996), 95-118. MR 97e:58199

[P5] - Iterations of holomorphic Collet-Eckmann maps: conformal and invariant measures, Trans. AMS 350.2 (1998), 717-742. MR 98d:58155

[P6] Hölder implies CE, Asterisque (to appear).

[PUbook] F. Przytycki, M. Urbański., Cambridge University Press (to appear).

[PUZ] F. Przytycki, M. Urbański, A. Zdunik, Harmonic, Gibbs and Hausdorff measures for holomorphic maps. I, Ann. of Math. 130 (1989), 1-40; II, Studia Math. 97 (1991), 189-225. MR 91i:58115; MR 93d:58140

[PR] F. Przytycki, S. Rohde, Porosity of Collet-Eckmann Julia sets, Fund. Math 155 (1998), 189-199. CMP 98:08

[R] D. Ruelle, An inequality for the entropy of differentiable maps, Bol. Soc. Bras. Mat. 9 (1978), 83-88. MR 80f:58126

[Shi] M. Shishikura, The Hausdorff dimension of the boundary of the Mandelbrot set and Julia sets, Ann. of Math. 147 (1998), 225-267. CMP 98:13

[Stein] E. Stein, Singular Integrals and Differentiability Properties of Functions, Princeton University Press, 1970. MR 44:7280

[S1] D. Sullivan, The density at infinity of a discrete group of hyperbolic motions, Publ. Math. IHES 50 (1979), 171-202. MR 81b:58031

[S2] _ Conformal dynamical systems, Geometric Dynamics, Lect. Notes in Math. 1007, Springer, 1983, pp. 725-752. MR 85m:58112 
[U1] M. Urbański, Rational functions with no recurrent critical points, Ergodic Th. and Dyn. Sys. 14.2 (1994), 391-414. MR 95g:58191

[U2] - On some aspects of fractal dimensions in higher dimensional dynamics, Proceedings of Workshop: Problems in higher dimensional dynamics., Preprint SFB 170 Goettingen, vol. 3, 1995, pp. 18-25.

[W] P. Walters, An Introduction to Ergodic Theory, Springer, 1982. MR 84e:28017

Institute of Mathematics, Polish Academy of Sciences, ul. Śniadeckich 8, 00950 WARSAW, POLAND

E-mail address: feliksp@impan.gov.pl 